IAU Colloquium 164: Radio Emission from Galactic and Extragalactic Compact Sources

ASP Conference Series, Vol. 144, 1998

J. A. Zensus, G. B. Taylor, \& J. M. Wrobel (eds.)

\title{
VLBA Observations of GRS 1915+105
}

V. Dhawan

National Radio Astronomy Observatory, Socorro, NM 87801, U.S.A.

I. F. Mirabel

Service d'Astrophysique, Saclay, F-91191 Gif-sur-Yvette, France.

L. F. Rodríguez

Instituto de Astronomia, UNAM, postal 70-264, 04510, Mexico

Abstract. During quasi-periodic flux variations, the core of this galactic superluminal source shows: (a) a flat radio spectrum between $13 \mathrm{~cm}$ and $2 \mathrm{~cm}$; (b) elongation of the core along the axis of arcsecond-scale ejecta; (c) a time-delay of $\sim 4$ mins at $3.6 \mathrm{~cm}$, relative to $2 \mathrm{~cm}$; (d) progressively less variation of the flux at $2 \mathrm{~cm}, 3.6 \mathrm{~cm} \& 13 \mathrm{~cm}$; and (e) scatter-broadening to 1.9 mas at $8.4 \mathrm{GHz}(135 \mathrm{mas}$ at $1 \mathrm{GHz})$.

GRS $1915+105$ is an X-ray binary discovered in 1992 by the Granat satellite (Castro-Tirado et al. 1994). VLA monitoring of a flux outburst in 1994 showed ejecta separating by $26 \mathrm{mas} /$ day, fit by an ejection velocity of $0.92 \mathrm{c}$ at $70^{\circ}$ to the line of sight (Mirabel \& Rodríguez 1994). A distance of $12.5 \mathrm{kpc}$ was derived from the $\mathrm{H} \mathrm{I}$ absorption (Rodríguez et al. 1995).

Radio emission from GRS 1915 has 2 states, steady (quasi-periodic) 20-100 mJy with an optically thick core; and flaring, when optically thin plasmoids are ejected. VLBI has been attempted six times, triggered by radio flares. No extended jets have been detected, probably because of their rapid expansion. However, in May and August 1996, we successfully used phase-referencing to image the core, which is elongated with major axis $\left(22^{\circ} \mathrm{W}\right.$ of $\left.\mathrm{N}\right)$, aligned with the arcsecond-scale ejecta. We take this as evidence for a quiescent jet.

The minor axis size scales as $\lambda^{2}$, interpreted as galactic scattering (135 mas at $1 \mathrm{GHz}$, at $\mathrm{l}=45.37, \mathrm{~b}=-0.22, \mathrm{D}=12.5 \mathrm{kpc}$ ). The scattering is enhanced and clumped on scales of $\sim 50 \mathrm{pc}$, by comparison with the $\mathrm{OH}$ maser in $\mathrm{G} 45.5+0.05$ (8.4 mas at $1 \mathrm{GHz}$, Diamond et al. 1988).

Flux oscillations in GRS 1915 have been previously modeled by an orbiting hot-spot of $\mathrm{T}_{B} \sim 10^{16} \mathrm{~K}$, embedded in absorbing plasma, (Rodríguez \& Mirabel 1997). New data (Fig.1) show that the variability is less at longer wavelength, contrary to absorption by a plasma. Here, we propose that a more conventional model, (e.g., Hjellming \& Johnston 1988), with incoherent synchrotron emission $\left(\mathrm{T}_{B} \sim 10^{11} \mathrm{~K}\right)$ in an optically thick jet of size $20-100 \mathrm{AU}(2.5-15$ mas), inclined to the line of sight, may account in a unified way for (a) the flat spectrum; (b) the elongation along the axis of ejection; (c) the time delay by geometry, as longer wavelengths emanate from further along the expanding jet; and (d) less variability at longer wavelengths, due to convolution over a larger region.

The radio variations may be associated with $\mathrm{x}$-ray bursts of the same period, corresponding to activity in the accretion disk at $\sim 1$ light second radius, $\sim 30$ minute Keplerian orbit.

Acknowledgments. The National Radio Astronomy Observatory is a facility of the NSF, operated under a cooperative agreement by Associated Universities, Inc. 


\section{References}

Castro-Tirado, A. J. et al., 1994. ApJS, 92, 469-472.

Diamond, P. J. et al. 1988. Radio Wave Scattering in the Interstellar Medium, eds. J. M. Cordes, B. J. Rickett, \& D. C. Backer, (New York: AIP), 174, 195-198.

Hjellming, R. M., \& Johnston, K. J. 1988. ApJ, 328, 600-609.

Mirabel, I. F., \& Rodriguez, L. F. 1994. Nature, 371, 46-48.

Rodriguez, L. F. et al. 1995. ApJS, 101, 173-179.

Rodríguez, L. F., \& Mirabel, I. F. 1997. ApJ, 474, L123-126.
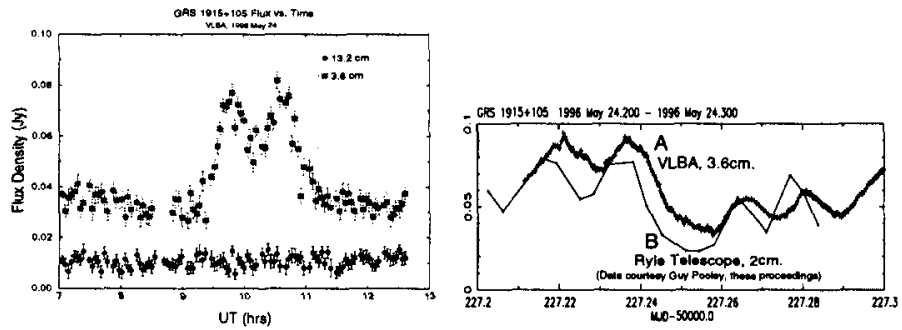

Figure 1. Left: Simultaneous light curves at $13.2 \mathrm{~cm}$, and $3.6 \mathrm{~cm}$, (raised by $20 \mathrm{~m} J \mathrm{y}$ ) Note the $30 \mathrm{~min}$ risetime at $3.6 \mathrm{~cm}$, and the lack of variation at $13.2 \mathrm{~cm}$. Right:Note $\sim 4$ minute time delay and reduced variation for the $3.6 \mathrm{~cm}$ light curve, compared to $2 \mathrm{~cm}$. ( $2 \mathrm{~cm}$ data courtesy Pooley \& Fender, these Proceedings, p. 333). The $\sim 40$ minute period is often seen in the X-rays and $2 \mathrm{~cm}$ emission, though simultaneous $\mathrm{X}$-ray data are not available.

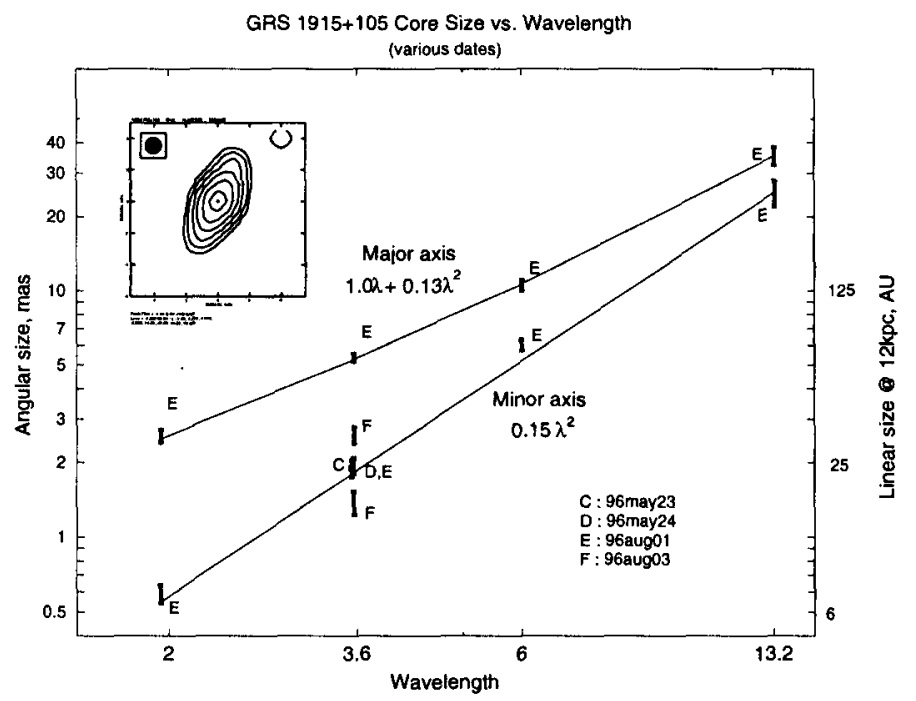

Figure 2. Deconvolved core size vs. wavelength, showing galactic scattering along the minor axis, and intrinsic elongation plus scattering of the major axis. Inset is the image at $2 \mathrm{~cm}$, restored with the $1 \times 1$ mas beam. Contours are $-2,2,4,8, \ldots, 96 \%$ of the peak $=65 \mathrm{mJy} /$ beam, and ticks are spaced at 2 mas. 